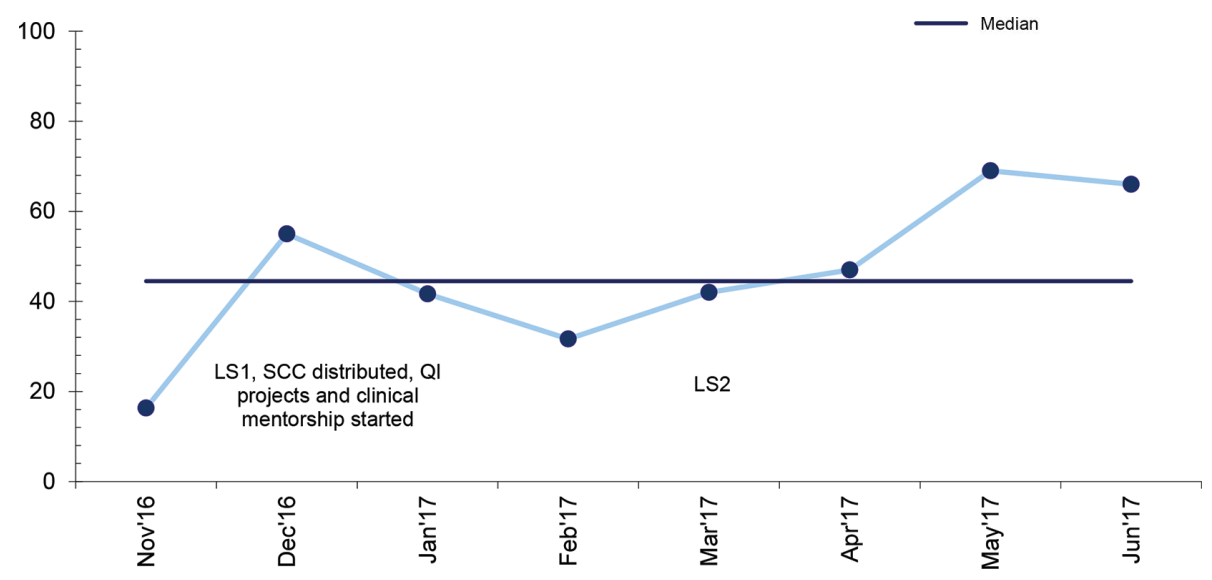

Abstract 865 Figure $6 \quad 100 \%$ completion of 'before pushing' bundle

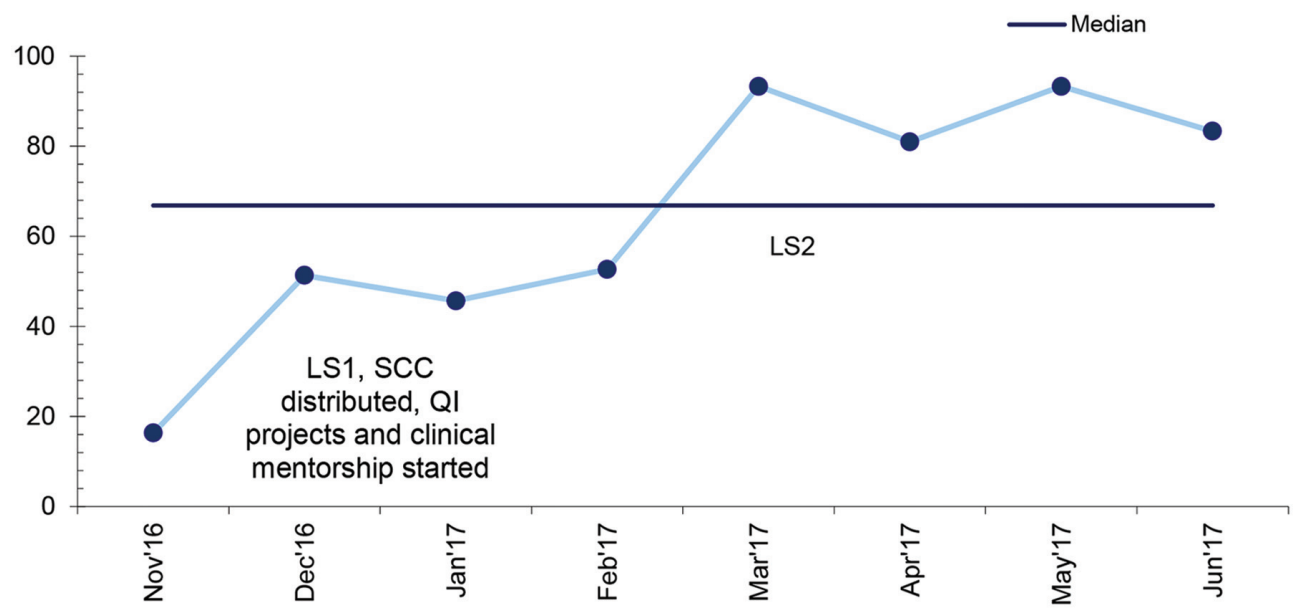

Abstract 865 Figure $7 \quad 100 \%$ completion of 'soon after birth' bundle

Conclusions Initial results are promising regarding program delivery and indicate high levels of facility-level QI activities, as well as initial signals of improved data and service quality in key maternal newborn health services.

\section{USING SIX SIGMA TO REDUCE READMISSIONS IN ELECTIVE TOTAL HIP \& KNEE ARTHROPLASTY}

${ }^{1}$ Brandon Hill, ${ }^{1}$ Eva Pittman, ${ }^{1}$ Hannah Lanier, ${ }^{1}$ Karen Hines, ${ }^{1}$ Charmaine Lewis, ${ }^{2}$ Jack Bowling, ${ }^{1}$ Caitlin Dunn, ${ }^{1}$ RoseAnn Gosswein, ${ }^{1}$ Shirley Glockner, ${ }^{1}$ David Oehler, ${ }^{1}$ Pam Cumber, 'Alice Matthews. ${ }^{1}$ New Hanover Regional Medical Centre, US; ${ }^{2}$ Bowling Orthopaedics, US

\subsection{6/bmjoq-2017-IHI.5}

Background New Hanover Regional Medical Centre is a public, not for profit, teaching hospital that performs over 2000 total joint arthroplasties per year. Following a near 1 million dollar penalty for excess 30 day Hip and Knee readmissions on the FY'15 CMS readmission report, a team was assembled to improve outcomes.

Objectives The team's objective was to identify trends among readmitted patients, conduct root cause analysis to determine process defects, identify risk factors within the population, optimise patients preoperatively, institute a risk scoring system to focus more energy and time with riskier patients, and decrease clinical variation that could lead to complications.

Methods Patient encounter data was used to conduct statistical analysis of medical diagnoses and demographic information to create a preoperative risk tool used to screen patients in preadmission testing. Risky patients were optimised, and received

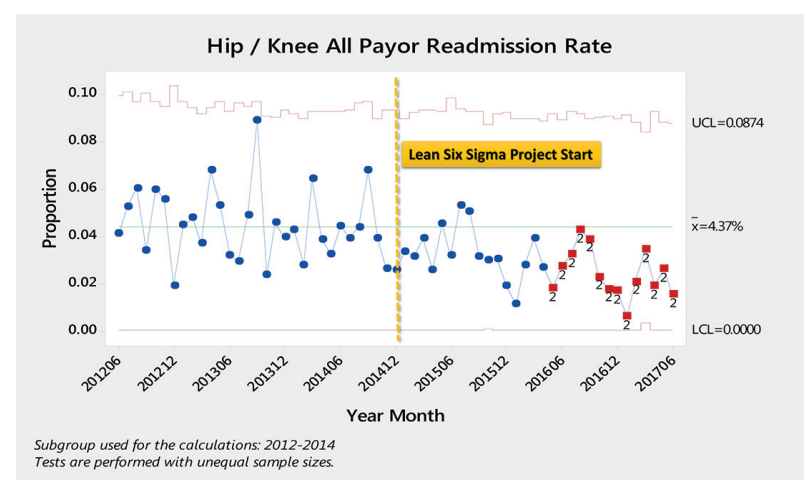

Abstract 869 Figure 1 Hip/knee all payor readmission rate 


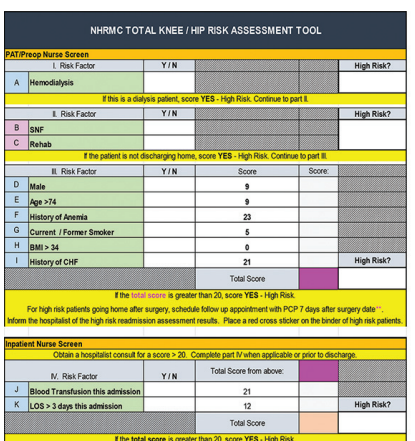

\begin{tabular}{|c|c|c|}
\hline Rootcalseffator & PValle & $\begin{array}{l}\text { Sigificantornot } \\
\text { 90\% Confidence enterval }\end{array}$ \\
\hline Gender & .004 & Yes Nales $1.5 \mathrm{X}$ more likely. \\
\hline Surgeon & .462 & No \\
\hline Age 74 Y Years & .000 & Yes 1.8 X more likely \\
\hline Race & .387 & No \\
\hline Discharge Dispo:SNF & .000 & Yes, SNF $1.8 \mathrm{BX}$ more litely \\
\hline Blood Transtusion & .000 & Yes, $3.7 \mathrm{X}$ more likely \\
\hline $\operatorname{los}>3$ days & .000 & res Los 302.25 more likely. \\
\hline CHF & .000 & Yes, $3.8 \mathrm{x}$ more likely \\
\hline Obesity(3MM / 35) & .308 & No \\
\hline Aremia & .000 & Yes Anemic 3 X morel lively \\
\hline Dibbetic & .011 & Yes $1.5 \mathrm{~m}$ more likey \\
\hline Strole & .014 & Yes, 2 more ilikely \\
\hline Renalfalure & .000 & Yes 2X more liely \\
\hline Tobaccoulse & .127 & No. Consider data reliability \\
\hline
\end{tabular}

Abstract 869 Figure 2 NHRMC total knee/hip risk assessment tool

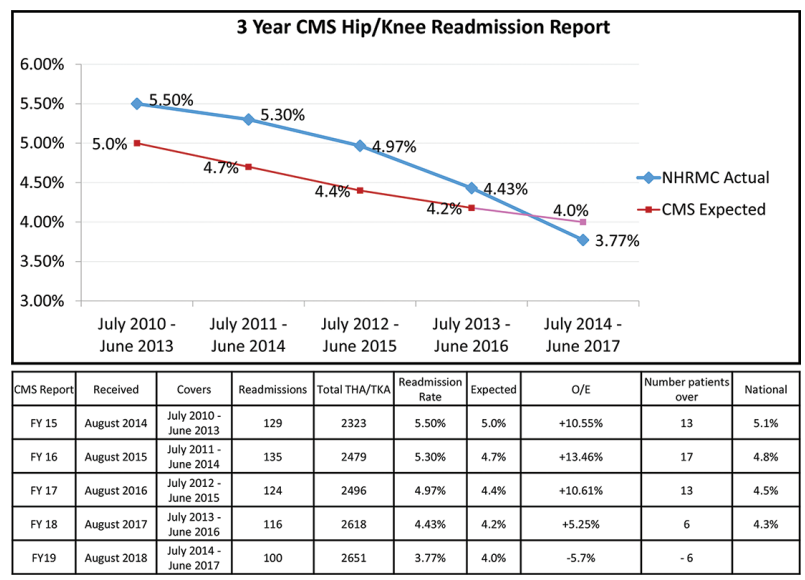

Abstract 869 Figure 33 year CMS hip/knee readmission report

more contact post discharge via phone calls focused on identified risk factors and common readmission reasons.

Results Initial CMS 3 year aggregate readmission rate was $5.5 \%$ versus $4.43 \% \mathrm{p}=0.04$ FY18 CMS report and $3.77 \%$ $\mathrm{p}=0.002$ FY'19 projected. Risk factor analysis and mirrored intervention strategies by other readmission populations and increased desire and utilisation of orthopaedic preadmission testing optimisation and anaemia clinic by other surgical populations.
Conclusions New Hanover Regional Medical Centre has statistically reduced readmissions and should receive no further Hip/Knee Arthroplasty Readmission penalties. Six sigma process of patient level analysis, statistically validated risk scoring, patient optimisation, reduced clinical variation, and risk based interventions can be utilised anywhere, with any population, to prevent unwanted outcomes.

\section{CONTINUOUS INFUSION MEDICATION SAFETY IN A PAEDIATRIC INTENSIVE CARE UNIT}

Ashley Ried. Monroe Carell Jr. Children's Hospital at Vanderbilt, US

\subsection{6/bmjoq-2017-IHI.6}

Background Failure to reliably use Computerised Physician Order Entry systems for continuous medications increases a patient's risk of experiencing adverse drug events, is outside the scope of practice for nurses and contradicts regulatory standards.

Objectives Increase the percentage of continuous medications infusing in the Paediatric Intensive Care Unit matching the current order from $74 \%$ to $90 \%$ by May 31, 2017 to eliminate adverse drug events.

Methods An interdisciplinary team conducted a Failure Modes Effects Analysis to identify failure modes and interventions. A Key Driver Diagram was then created to frame the improvement work. The first Plan-Do-Study-Act cycle ensured ordering providers read back orders on rounds to provide real-time loop closure. A Pareto chart of failures identified vasoactive medications as the largest non-compliant category, leading to another PDSA implementing vasoactive titration order-sets. Rapid cycle testing and feedback allowed the team to address concerns before full implementation.

Results Statistical process control was used for the percentage of continuous medications and vasopressors matching the provider order displayed in separate p-charts. Established rules were used for differentiating special versus common cause variation. 431 baseline observational audits revealed a mean compliance of $74 \%$ that increased to $93 \%$ after 12 months. Subset analysis of vasoactive medications showed an improvement from $38 \%$ to $89 \%$ compliance.

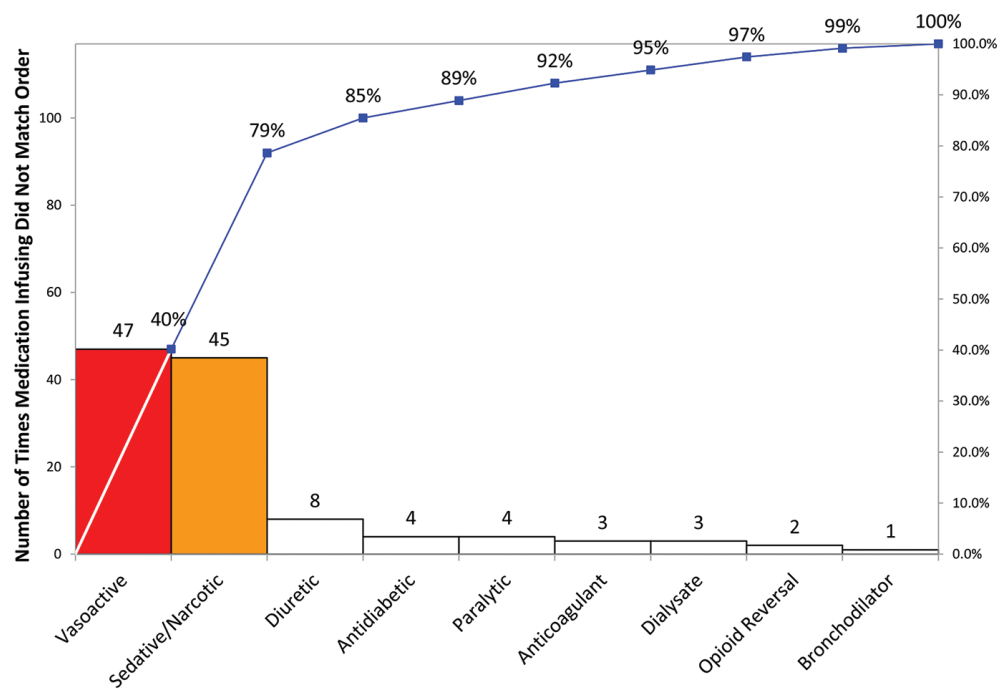

Abstract 873 Figure 1 Pareto: PICU continuous IV medication infusions by class that do not match order 6/5/16-9/27/16 